\title{
The greatest prime divisor of a product of consecutive integers
}

\author{
by \\ Shanta Laishram and T. N. Shorey (Mumbai)
}

1. Introduction. Let $k \geq 2$ and $n \geq 1$ be integers. We define

$$
\Delta(n, k)=n(n+1) \cdots(n+k-1) .
$$

For an integer $\nu>1$, we denote by $\omega(\nu)$ and $P(\nu)$ the number of distinct prime divisors of $\nu$ and the greatest prime factor of $\nu$, respectively, and we put $\omega(1)=0, P(1)=1$.

A well known theorem of Sylvester [7] states that

$$
P(\Delta(n, k))>k \quad \text { if } n>k .
$$

We observe that $P(\Delta(1, k)) \leq k$ and therefore the assumption $n>k$ in (1) cannot be removed. For $n>k$, Moser [5] sharpened (1) to $P(\Delta(n, k))>\frac{11}{10} k$ and Hanson [3] to $P(\Delta(n, k))>1.5 k$ unless $(n, k)=(3,2),(8,2),(6,5)$. Further Faulkner [2] proved that $P(\Delta(n, k))>2 k$ if $n$ is greater than or equal to the least prime exceeding $2 k$ and $(n, k) \neq(8,2),(8,3)$.

In this paper, we sharpen the results of Hanson and Faulkner. We shall not use these results in the proofs of our improvements. We prove

Theorem 1. We have

(a)

$$
P(\Delta(n, k))>2 k \quad \text { for } n>\max \left(k+13, \frac{279}{262} k\right) .
$$

(b)

$$
P(\Delta(n, k))>1.97 k \quad \text { for } n>k+13 .
$$

We observe that 1.97 in (3) cannot be replaced by 2 since there are arbitrarily long chains of consecutive composite positive integers. The same reason implies that Theorem 1(a) is not valid under the assumption $n>$

2000 Mathematics Subject Classification: Primary 11A41, 11N05, 11N13.

Key words and phrases: arithmetic progressions, primes. 
$k+13$. Further the assumption $n>\frac{279}{262} k$ in Theorem 1 (a) is necessary since $P(\Delta(279,262)) \leq 2 \cdot 262$.

Now we give a lower bound for $P(\Delta(n, k))$ which is valid for $n>k>2$ except for an explicitly given finite set. For this, we need some notation. For a pair $(n, k)$ and a positive integer $h$, we write $[n, k, h]$ for the set of all pairs $(n, k), \ldots,(n+h-1, k)$ and we set $[n, k]=[n, k, 1]=\{(n, k)\}$. Let

$$
\begin{aligned}
& A_{10}=\{58\}, \quad A_{8}=A_{10} \cup\{59\}, \quad A_{6}=A_{8} \cup\{60\}, \\
& A_{4}=A_{6} \cup\{12,16,46,61,72,93,103,109,151,163\}, \\
& A_{2}=A_{4} \cup\{4,7,10,13,17,19,25,28,32,38,43,47,
\end{aligned}
$$

and $A_{2 i+1}=A_{2 i}$ for $1 \leq i \leq 5$. Further let

$$
\begin{array}{r}
A_{1}=A_{2} \cup\{3,5,6,8,9,11,14,15,18,20,23,26,29,33,35,39,41,44,48,50,53, \\
56,63,68,74,78,81,86,89,95,105,111,125,146,153,165,173,270\} .
\end{array}
$$

Finally, we set

$$
B=[8,3] \cup[5,4,3] \cup[14,13,3] \cup\{(k+1, k) \mid k=3,5,8,11,14,18,63\} .
$$

Then

THEOREM 2. We have

$$
P(\Delta(n, k))>1.95 k \quad \text { for } n>k>2
$$

except when $(n, k) \in[k+1, k, h]$ for $k \in A_{h}$ with $1 \leq h \leq 11$ or $(n, k)=$ $(8,3)$.

If $k=2$, we observe (see Lemma 7$)$ that $P(\Delta(n, k))>2 k$ unless $n=3,8$ and that $P(\Delta(3,2))=P(\Delta(8,2))=3$. Thus the estimate $(4)$ is valid for $k=2$ whenever $n \neq 3,8$. We observe that $P(\Delta(k+1, k)) \leq 2 k$ and therefore 1.95 in (4) cannot be replaced by 2 .

There are few exceptions if 1.95 is replaced by 1.8 in Theorem 2. We derive from Theorem 2 the following result.

Corollary 1. We have

$$
P(\Delta(n, k))>1.8 k \quad \text { for } n>k>2
$$

except when $(n, k) \in B$.

2. Lemmas. We begin with a well known result due to Levi ben Gerson on a particular case of the Catalan equation.

Lemma 1. The solutions of

$$
2^{a}-3^{b}= \pm 1 \quad \text { in integers } a>0, b>0
$$

are given by $(a, b)=(1,1),(2,1),(3,2)$. 
Next we state a result of Saradha and Shorey [6] on a lower bound for $\omega(\Delta(n, k))$.

Lemma 2. For $n>k>2$, we have

$$
\omega(\Delta(n, k)) \geq \pi(k)+\left[\frac{1}{3} \pi(k)\right]+2
$$

except when $(n, k)$ belongs to the union of the sets

$$
\left\{\begin{array}{l}
{[4,3],[6,3,3],[16,3],[6,4],[6,5,4],[12,5],[14,5,3],[23,5,2],} \\
{[7,6,2],[15,6],[8,7,3],[12,7],[14,7,2],[24,7],[9,8],[14,8],} \\
{[14,13,3],[18,13],[20,13,2],[24,13],[15,14],[20,14],[20,17] .}
\end{array}\right.
$$

We shall use Lemma 2 only when $k=3$ or $5 \leq k \leq 8$. Let $p_{i}$ denote the $i$ th prime number. Then

Lemma 3. We have

(6)

$$
p_{i+1}-p_{i}< \begin{cases}35 & \text { for } p_{i} \leq 5591, \\ 15 & \text { for } p_{i} \leq 1123, p_{i} \neq 523,887,1069, \\ 21 & \text { for } p_{i}=523,887,1069, \\ 9 \quad & \text { for } p_{i} \leq 361, \\ & p_{i} \neq 113,139,181,199,211,241,283,293,317,337 .\end{cases}
$$

Lemma 4. Let $\mathfrak{N}$ be a positive real number and $k_{0}$ a positive integer. Let $I\left(\mathfrak{N}, k_{0}\right)=\left\{i \mid p_{i+1}-p_{i} \geq k_{0}, p_{i} \leq \mathfrak{N}\right\}$. Then

$$
P(n(n+1) \cdots(n+k-1))>2 k
$$

for $2 k \leq n<\mathfrak{N}$ and $k \geq k_{0}$ except possibly when $p_{i}<n<n+k-1<p_{i+1}$ for $i \in I\left(\mathfrak{N}, k_{0}\right)$.

Proof. Let $2 k \leq n<\mathfrak{N}$ and $k>k_{0}$. We may suppose that none of $n, n+1, \ldots, n+k-1$ is a prime, otherwise the result follows. Let $p_{i}<n<$ $n+k-1<p_{i+1}$. Then $i=\pi(n)$ and $p_{\pi(n)}<n<\mathfrak{N}$. For $\pi(n) \notin I\left(\mathfrak{N}, k_{0}\right)$, we have

$$
k-1=n+k-1-n<p_{\pi(n)+1}-p_{\pi(n)}<k_{0},
$$

which implies $k-1<k_{0}-1$, a contradiction. Hence the assertion.

The following result on the estimates for primes is due to Dusart [1, p. 14].

Lemma 5. For $\nu>1$, we have
(i) $\pi(\nu) \leq \frac{\nu}{\log \nu}\left(1+\frac{1.2762}{\log \nu}\right)$,
(ii) $\pi(\nu) \geq \frac{\nu}{\log \nu-1} \quad$ for $\nu \geq 5393$. 
Lemma 6. Let $X>0$ and $0<\theta<e-1$ be real numbers. For $l \geq 0$, let

$$
\begin{aligned}
X_{0} & =\max \left(\frac{5393}{1+\theta}, \exp \left(\frac{\log (1+\theta)+0.2762}{\theta}\right)\right), \\
X_{l+1} & =\max \left(\frac{5393}{1+\theta}, \exp \left(\frac{\log (1+\theta)+0.2762}{\theta+\frac{1.2762(1-\log (1+\theta))}{\log ^{2} X_{l}}}\right)\right) .
\end{aligned}
$$

Then

$$
\pi((1+\theta) X)-\pi(X)>0 \quad \text { for } X>X_{l} .
$$

Proof. Let $l \geq 0$ and $X>X_{l}$. Then $(1+\theta) X \geq 5393$. By Lemma 5 , we have

$$
\begin{aligned}
\delta & :=\pi((1+\theta) X)-\pi(X) \geq \frac{(1+\theta) X}{\log (1+\theta) X-1}-\frac{X}{\log X}\left(1+\frac{1.2762}{\log X}\right) \\
& \geq \frac{X}{\log (1+\theta) X-1}\left\{1+\theta-\frac{\log (1+\theta) X-1}{\log X}\left(1+\frac{1.2762}{\log X}\right)\right\} \\
& \geq \frac{X}{\log (1+\theta) X-1}\left\{1+\theta-\left(1-\frac{1-\log (1+\theta)}{\log X}\right)\left(1+\frac{1.2762}{\log X}\right)\right\} \\
& \geq \frac{X}{\log (1+\theta) X-1}\{F(X)+G(X)\}
\end{aligned}
$$

where

$$
F(X)=\theta-\frac{\log (1+\theta)+0.2762}{\log X}, \quad G(X)=\frac{1.2762(1-\log (1+\theta))}{\log ^{2} X} .
$$

We see that $G(X)>0$ and is decreasing since $0<\theta<e-1$. Further we observe that $\left\{X_{i}\right\}$ is a non-increasing sequence. We notice that $\delta>0$ if $F(X)+G(X)>0$. But $F(X)+G(X)>F(X)>0$ for $X>X_{0}$ by the definition of $X_{0}$. Thus $\delta>0$ for $X>X_{0}$.

Let now $X \leq X_{0}$. Then $F(X)+G(X) \geq F(X)+G\left(X_{0}\right)$ and $F(X)+$ $G\left(X_{0}\right)>0$ if $X>X_{1}$ by the definition of $X_{1}$. Hence $\delta>0$ for $X>X_{1}$. Now we proceed inductively as above to see that $\delta>0$ for $X>X_{l}$ with $l \geq 2$.

Lemma 7. Let $n>k$ and $k \leq 16$. Then

$$
P(\Delta(n, k)) \leq 2 k
$$

implies that $(n, k) \in\{(8,2),(8,3)\}$ or $(n, k) \in[k+1, k]$ for $k \in\{2,3,5,6,8$, $9,11,14,15\}$ or $(n, k) \in[k+1, k, 3]$ for $k \in\{4,7,10,13\}$ or $(n, k) \in[k+1$, $k, 5]$ for $k \in\{12,16\}$.

Proof. We apply Lemma 1 to derive that (7) is possible only if $n=3,8$ when $k=2$ and $n=5,6,7$ when $k=4$. For the latter assertion, we apply Lemma 1 after securing $P((n+i)(n+j)) \leq 3$ with $0 \leq i<j \leq 3$ by deleting the terms divisible by 5 and 7 in $n, n+1, n+2$ and $n+3$. For $k=3$ and $5 \leq k \leq 8$, the assertion follows from Lemma 2 . 
Thus we may assume that $k \geq 9$. Assume that (7) holds. Then in the product $\Delta(n, k)$, there are at most $1+[(k-1) / p]$ terms divisible by the prime $p$. After removing all the terms divisible by $p \geq 7$, we are left with at least four terms only divisible by 2,3 and 5 . Further out of these terms, for each prime 2,3 and 5 , we remove a term in which the prime divides to a maximal power. Then we are left with a term $n+i$ such that $n \leq n+i \leq$ $8 \cdot 9 \cdot 5=360$.

Let $n \geq 2 k$. We now apply Lemma 4 with $\mathfrak{N}=361, k_{0}=9$ and (6) to get $P(\Delta(n, k))>2 k$ for $k \geq 9$ except possibly when $p_{i}<n<n+k-1<p_{i+1}$, $p_{i}=113,139,181,199,211,241,283,293,317,337$. For these values of $n$, we check that $P(\Delta(n, k))>2 k$ is valid for $9 \leq k \leq 16$. Thus it suffices to consider $k<n<2 k$. We calculate $P(\Delta(n, k))$ for $(n, k)$ with $9 \leq k \leq 16$ and $k<n<2 k$. We find that (7) holds only if $(n, k)$ is as given in the statement of Lemma 7.

3. Proof of Theorem 1(a). Let $n>\max \left(k+13, \frac{279}{262} k\right)$. In view of Lemma 7 , we may take $k \geq 17$ since $n \leq k+5$ for the exceptions $(n, k)$ given in Lemma 7 . It suffices to prove (2) for $k$ such that $2 k-1$ is prime. Let $k_{1}<k_{2}$ be such that $2 k_{1}-1$ and $2 k_{2}-1$ are consecutive primes. Suppose (2) holds at $k_{1}$. Then for $k_{1}<k<k_{2}$, we have

$$
P(n(n+1) \cdots(n+k-1)) \geq P\left(n \cdots\left(n+k_{1}-1\right)\right)>2 k_{1},
$$

implying $P(\Delta(n, k)) \geq 2 k_{2}-1>2 k$. Therefore we may suppose that $k \geq 19$ since $2 k-1$ with $k=17,18$ are composites. We assume from now onward in the proof of Theorem 1 (a) that $2 k-1$ is prime. We put $x=n+k-1$. Then $\Delta(n, k)=x(x-1) \cdots(x-k+1)$. Let $f_{1}<\cdots<f_{\mu}$ be all the integers in $[0, k)$ such that

$$
P\left(\left(x-f_{1}\right) \cdots\left(x-f_{\mu}\right)\right) \leq k .
$$

We argue as in the proof of $[4$, Lemma 4$]$ to get

$$
k !>x^{\mu-\pi(k)}\left(1-\frac{k}{x}\right)^{\mu} .
$$

We may suppose $\omega(\Delta(n, k)) \leq \pi(2 k)$, otherwise (2) follows. Then

$$
\mu \geq k-\pi(2 k)+\pi(k)
$$

which we use as in [4, Lemma 4] to derive from (9) that

$$
x<k^{3 / 2} \text { for } k \geq 87 ; \quad x<k^{7 / 4} \text { for } k \geq 40 ; \quad x<k^{2} \text { for } k \geq 19 .
$$

If $x \geq 7 k$ and $k>57$, then as in [4, Lemma 7] we derive from (10) that $x \geq$ $k^{3 / 2}$. Thus (11) implies that $x<7 k$ for $k \geq 87$. Putting back $n=x-k+1$, we may assume that $n<6 k+1$ for $k \geq 87, n<k^{7 / 4}-k+1$ for $40 \leq k<87$ and $n<k^{2}-k+1$ for $19 \leq k<40$. 
Let $k<87$. Suppose $n \geq 2 k$. Then $2 k \leq n<k^{7 / 4}-k+1$ for $40 \leq k<87$ and $2 k \leq n<k^{2}-k+1$ for $19 \leq k<40$. Thus Lemma 4 with $\mathfrak{N}=$ $87^{7 / 4}-87+1, k_{0}=35$ and (6) implies that $P(\Delta(n, k))>2 k$ for $k \geq 35$. We note here that $2 k \leq n<\mathfrak{N}$ for $35 \leq k<40$. Let $k<35$. Taking $\mathfrak{N}=$ $34^{2}-34+1, k_{0}=21$ for $21 \leq k \leq 34$ and $\mathfrak{N}=19^{2}-19+1, k_{0}=19$ for $k=19$, we see from Lemma 4 and (6) that $P(\Delta(n, k))>2 k$ for $k \geq 19$. Here the case $k=20$ is excluded since $2 k-1$ is composite. Therefore we may assume that $n<2 k$. Further we observe that $\pi(n+k-1)-\pi(2 k) \geq \pi(2 k+13)-\pi(2 k)$ since $n>k+13$. Next we check that $\pi(2 k+13)-\pi(2 k)>0$. This implies that $[2 k, n+k-1]$ contains a prime.

Thus we may assume that $k \geq 87$. Then we write

$$
n=\alpha k+1 \quad \text { with } \begin{cases}279 / 262-1 / k<\alpha \leq 6 & \text { if } k \geq 201 \\ 1+12 / k<\alpha \leq 6 & \text { if } k<201\end{cases}
$$

Further we consider $\pi(n+k-1)-\pi(\max (n-1,2 k))$, which is

$$
\begin{array}{ll}
=\pi((\alpha+1) k)-\pi(\alpha k) & \text { for } \alpha \geq 2, \\
\geq \pi\left(\left[\frac{541}{262} k\right]\right)-\pi(2 k) & \text { for } \alpha<2 \text { and } k \geq 201, \\
\geq \pi(2 k+13)-\pi(2 k) & \text { for } \alpha<2 \text { and } k<201 .
\end{array}
$$

By using exact values of the $\pi$ function we check that

$$
\begin{aligned}
\pi(2 k+13)-\pi(2 k)>0 & \text { for } k<201, \\
\pi\left(\left[\frac{541}{262} k\right]\right)-\pi(2 k)>0 & \text { for } 201 \leq k \leq 2616 .
\end{aligned}
$$

Thus we may suppose that $k>2616$ if $\alpha<2$. Also

$$
\left[\frac{541}{262} k\right] \geq \frac{540}{262} k \quad \text { for } k>2616 .
$$

Now we apply Lemma 6 with $X=\alpha k, \theta=1 / \alpha, l=0$ if $\alpha \geq 2$ and $X=$ $2 k, \theta=4 / 131, l=1$ if $\alpha<2$ to get

$$
\pi(n+k-1)-\pi(\max (n-1,2 k))>0
$$

for $X>X_{0}=5393 /(1+1 / \alpha)$ if $\alpha \geq 2$ and $X>X_{1}=5393 /(1+4 / 131)$ if $\alpha<2$. Further when $\alpha<2$, we observe that $X=2 k>X_{1}$ since $k>2616$. Thus the assertion follows for $n<2 k$.

It remains to consider the case $\alpha \geq 2$ and $X \leq 5393(1+1 / \alpha)^{-1}$. Then $2 k \leq n<n+k-1=X(1+1 / \alpha) \leq 5393$. Now we apply Lemma 4 with $\mathfrak{N}=5393, k_{0}=35$ and (6) to conclude that $P(\Delta(n, k))>2 k$.

4. Proof of Theorem 1(b). In view of Lemma 7 and Theorem 1(a), we may assume that $k \geq 17$ and $k<n \leq \frac{279}{262} k$. Let $X=\frac{279}{262} k, \theta=\frac{245}{279}, l=0$. 
Then for $k<n \leq X$, we see from Lemma 6 that

$$
\pi(2 k)-\pi(n-1) \geq \pi((1+\theta) X)-\pi(X)>0
$$

for $X>X_{0}=5393(1+\theta)^{-1}$ which is satisfied for $k>2696$ since $(1+\theta) X=$ $2 k$. Thus we may suppose that $k \leq 2696$. Now we check with exact values of the $\pi$ function that $\pi(2 k)-\pi\left(\frac{2 \overline{7} 9}{262} k\right)>0$. Therefore

$$
P(\Delta(n, k)) \geq P(n(n+1) \cdots 2 k) \geq p_{\pi(2 k)} .
$$

Further we apply Lemma 6 with $X=1.97 k, \theta=3 / 197$ and $l=25$. We calculate that $X_{l} \leq 284000$. We conclude by Lemma 6 that

$$
\pi(2 k)-\pi(1.97 k)=\pi((1+\theta) X)-\pi(X)>0
$$

for $k>145000$. Let $k \leq 145000$. Then we check that $\pi(2 k)-\pi(1.97 k)>0$ is valid for $k \geq 680$ by using exact values of the $\pi$ function. Thus

$$
p_{\pi(2 k)}>1.97 k \quad \text { for } k \geq 680 \text {. }
$$

Therefore we may suppose that $k<680$. Now we observe that for $n>k+13$,

$$
\pi(n+k-1)-\pi(1.97 k) \geq \pi(2 k+13)-\pi(1.97 k)>0 ;
$$

the latter inequality can be checked by using exact values of the $\pi$ function. Hence the assertion follows since $n<1.97 k$.

5. Proof of Theorem 2. By Theorem 1(b), we may assume that $n \leq$ $k+13$. Also we may suppose that $k<680$ by (12). For $k \leq 16$, we calculate $P(\Delta(n, k))$ for all the pairs $(n, k)$ given in the statement of Lemma 7 . We find that either $P(\Delta(n, k))>1.95 k$ or $(n, k)$ is an exception stated in Theorem 1 (a). Thus we may suppose that $k \geq 17$. Now we check that $\pi(n+k-1)-$ $\pi(1.95 k)>0$ except when $(n, k) \in[k+1, k, h]$ for $k \in A_{h}$ with $1 \leq h \leq 11$, and the assertion follows.

6. Proof of Corollary 1. We calculate $P(\Delta(n, k))$ for all $(n, k)$ with $k \leq 270$ and $k+1 \leq n \leq k+11$. This contains the set of exceptions given in Theorem 2. We find that $P(\Delta(n, k))>1.8 k$ unless $(n, k) \in B$. Hence the assertion (5) follows from Theorem 2.

\section{References}

[1] P. Dusart, Autour de la fonction qui compte le nombre de nombres premiers, Ph.D. thesis, Univ. de Limoges, 1998.

[2] M. Faulkner, On a theorem of Sylvester and Schur, J. London Math. Soc. 9 (1934), $282-288$.

[3] D. Hanson, On a theorem of Sylvester and Schur, Canad. Math. Bull. 16 (1973), 195-199.

[4] S. Laishram and T. N. Shorey, Number of prime divisors in a product of consecutive integers, Acta Arith. 113 (2004), 327-341. 
[5] L. Moser, Insolvability of $\left(\begin{array}{c}2 n \\ n\end{array}\right)=\left(\begin{array}{c}2 a \\ a\end{array}\right)\left(\begin{array}{c}2 b \\ b\end{array}\right)$, Canad. Math. Bull. (2) 6 (1963), 167-169.

[6] N. Saradha and T. N. Shorey, Almost squares and factorisations in consecutive integers, Compositio Math. 138 (2003), 113-124.

[7] J. J. Sylvester, On arithmetical series, Messenger of Mathematics 21 (1892), 1-19, 87-120, and Mathematical Papers, Vol. 4, 1912, 687-731.

School of Mathematics

Tata Institute of Fundamental Research

Homi Bhabha Road

Mumbai 400005, India

E-mail: shanta@math.tifr.res.in shorey@math.tifr.res.in

Received on 14.2.2005

and in revised form on 4.7.2005 